\title{
4 Genetic Variation in Phenotypic Plasticity
}

\author{
Ilan Goldstein and lan M. Ehrenreich \\ University of Southern California
}

\section{CONTENTS}

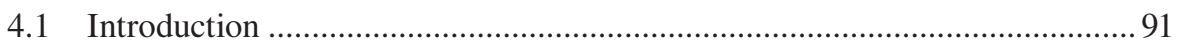

4.2 The Geneticist's View of Phenotypic Plasticity............................................92

4.3 Modification of Heritable Phenotypic Variation by the Environment.............94

4.4 Methods for Genetically Dissecting Phenotypic Plasticity .............................96

4.5 Empirical Insights into the Genetics of Phenotypic Plasticity ........................98

4.6 The Future of the Genetics of Phenotypic Plasticity .................................... 101

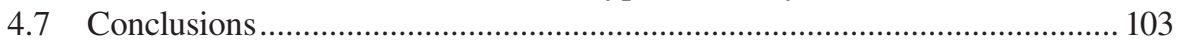

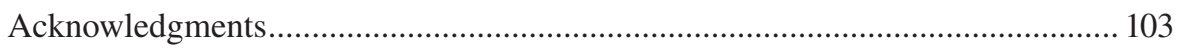

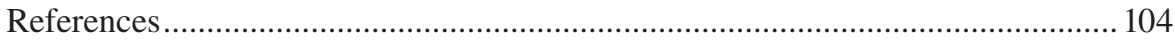

\subsection{INTRODUCTION}

Most traits of human interest vary among individuals within a population due to a combination of genetic and environmental factors, meaning individuals' phenotypes will depend on both their genotypes and environments. This relationship between genotype, environment, and phenotype is a major area of research that is central to our understanding of genetics in the real world (Falconer and Mackay 1996; Lynch and Walsh 1998). Indeed, whether the goal be to breed crops that maximize yield in particular climates (Kang 1997; Gage et al. 2017; Lowry et al. 2019), to predict, prevent, and treat hereditary disorders (Hunter 2005; Baye et al. 2011; Manuck and McCaffery 2014), or to understand the mechanisms underlying adaptation to changing environmental conditions (Bradshaw 1965; Via and Lande 1985; Scheiner 1993), all of these topics require knowledge of how genotype and environment jointly produce phenotype.

Scientists can learn about the relationship between genotype, environment, and phenotype by studying phenotypic plasticity, the ability of a genotype to produce different phenotypes in response to different environments (Bradshaw 1965; Scheiner 1993; Pigliucci 2001; West-Eberhard 2003). Within genetically diverse populations, distinct genotypes will commonly show differences in plasticity (Ehrenreich and Pfennig 2016). Geneticists dissect this heritable variation in plasticity in order to better determine the impact of environment on the relationship between individuals' genotypes and phenotypes (Debat and David 2001; Rockman 2008; Mackay et al. 2009). 
Here, we review genetic research on variation in plasticity. In addition to summarizing key concepts and methods, we also attempt to synthesize current, empirical work in this area and point to key future directions.

\subsection{THE GENETICIST'S VIEW OF PHENOTYPIC PLASTICITY}

Plasticity arises when the environment alters how physiological and developmental processes impact phenotype (Bradshaw 1965; Dufty Jr. et al. 2002; Schlichting and Smith 2002; Aubin-Horth and Renn 2009; Beldade et al. 2011; Lafuente and Beldade 2019). However, genetically distinct individuals may respond differently to the same environments (Bradshaw 1965; Via and Lande 1985; Scheiner 1993). This heritable variation in plasticity is caused by environmentally responsive genetic polymorphisms (or 'loci') that segregate among individuals (Falconer and Mackay 1996; Lynch and Walsh 1998). Individuals carrying distinct alleles of such loci will exhibit different phenotypic responses to the same conditions (Hunter 2005). Depending on the alleles present, the phenotypic differences between individuals that have distinct genotypes at an environmentally responsive locus may increase, decrease, or change in sign from one condition to another (Figure 4.1b). Because their effects depend on the environment under study, these loci are said to show 'gene-environment interactions' (Figure. 4.1a) (Matsui and Ehrenreich 2016; Wei and Zhang 2017; see also Sultan 2021 in this volume).

Populations usually harbor many loci that interact with the environment (Mackay 2001; Mackay et al. 2009). Thus, variability in plasticity among individuals must be viewed as a result of 'genotype-environment interaction'-i.e., interactions between the environment and individuals' entire complement of environmentally responsive loci (Lee et al. 2016; Matsui and Ehrenreich 2016). Each of these loci may contribute to an overall genotype-environment interaction either 'additively,' 'epistatically,' or both (Gerke et al. 2010; Lee et al. 2016, 2019; Matsui and Ehrenreich 2016; Wei and Zhang 2017). Additive loci have the same effects regardless of the alleles present at other loci (Figure 4.1c). Epistatic loci, on the other hand, participate in genetic interactions with other loci. Consequently, they show different effects depending on the alleles with which they co-occur (Figure 4.1d) (Mackay 2014; Ehrenreich 2017). Genotype-environment interactions may arise due to combinations of purely additive or epistatic loci, or a mixture of the two (Figure 4.1e) (Scheiner 1993; Gerke et al. 2010; Lee et al. 2016, 2019; Matsui and Ehrenreich 2016; Wei and Zhang 2017).

A complete characterization of genotype-environment interaction should also encompass information about the specific genes and genetic variants that contribute to plasticity (Mackay 2001; Mackay et al. 2009). Although most studies do not achieve this degree of precision, it is important to recognize that loci affecting traits represent genetic variants that alter how particular genes function (Schlichting and Pigliucci 1993). At the molecular level, this may involve genetic variants altering transcription, splicing, protein activity, or other aspects of gene function. Of course, the functional impact of a particular genetic variant will depend on the exact nature of the variant at a locus, as well as the identity of the specific gene directly affected by this variant (Gerke et al. 2010; Lee et al. 2016, 2019; Matsui and Ehrenreich 2016). 

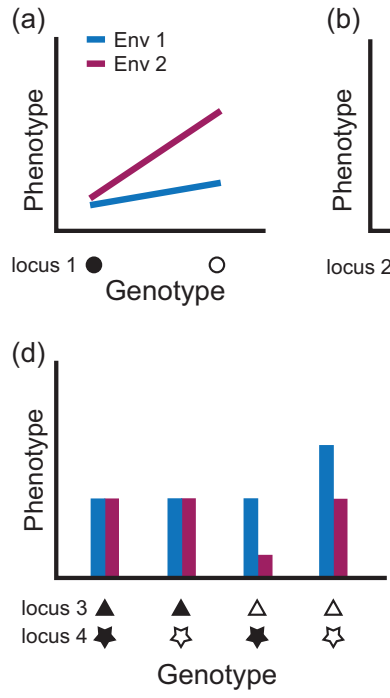

(b)

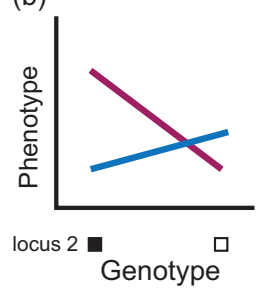

(e)

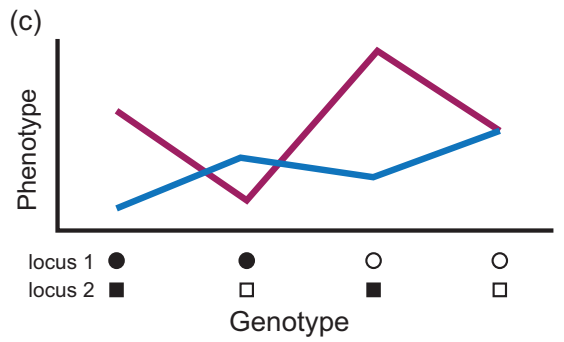

(c)

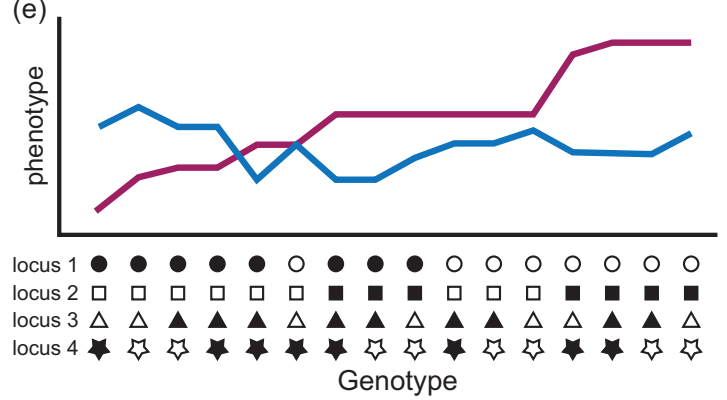

FIGURE 4.1 Examples of gene- and genotype-environment interactions. (a) Locus 1 shows a larger phenotypic effect in response to environment 2 than to environment 1 . (b) Locus 2 exhibits a change in effect sign across the two environments. (c) Loci 1 and 2 contribute additively to phenotype across environments. (d) Loci 3 and 4 contribute epistatically to environmental response. Their genetic interaction has a positive effect in environment 1 and a negative effect in environment 2. (e) The relationship between all four loci and phenotype across the two environments. Genotypes are sorted by the rank order of their phenotypes in environment 2. Note that the small effects of each involved locus lead to complicated genotype-phenotype relationships across environments, even within the simplified examples presented here. The different symbols represent distinct loci, while the filled and unfilled versions of each symbol correspond to the two different alleles of each locus.

Because genes usually act in pathways and networks, a genetic variant may also modify the functional relationships between the impact gene and other genes (Ayroles et al. 2009; Civelek and Lusis 2014). There are likely many different mechanisms that can cause loci to show gene-environment interaction, and the continued characterization of loci that cause plasticity can improve our comprehension of these mechanisms.

The above concepts collectively serve as the foundation for understanding the genetic architecture of plasticity. Here, genetic architecture refers to all loci involved in a plastic phenotypic response, as well as their individual effects and interactions with each other and the environment (Hansen 2006; Flint and Mackay 2009). For a given trait, a complete description of the genetic architecture of plasticity would provide a predictive map relating individuals' genotypes and environments to their phenotypes (Gerke et al. 2010; Lee et al. 2016, 2019; Matsui and Ehrenreich 2016; Wei and Zhang 2017). We discuss the methods involved in such work later in this manuscript. We note though, that for a variety of biological and technical reasons mentioned below, obtaining such a complete portrait is difficult in most organisms. 
However, large-scale studies in model organisms are beginning to achieve a comprehensive understanding of the genetic architecture of plasticity for certain traits.

\subsection{MODIFICATION OF HERITABLE PHENOTYPIC VARIATION BY THE ENVIRONMENT}

In this section, we discuss additional concepts that are often used to think about how the genotype-environment interactions that cause plasticity impact the amounts and patterns of heritable phenotypic variation within a population. 'Environmental robustness' - the ability of an organism to express the same phenotype despite environmental perturbation-is of particular importance (Kitano 2004; Felix and Barkoulas 2015). Plasticity and robustness represent opposite extremes of a spectrum, respectively corresponding to states of high and low environmental sensitivity (Figure 4.2) (Ehrenreich and Pfennig 2016; Nijhout et al. 2017; see also Pfennig 2021 in this volume). This view of plasticity and robustness as having an inverse relationship holds in many circumstances, but it is not universally true. For example, some species may evolve to be robustly plastic, e.g., it may be adaptive to express a specific polyphenism in response to particular, repeating environmental cues (Bateson and Gluckman 2011). While we acknowledge this special case, it is not our emphasis here.

Because most organisms live in dynamic environments, they have evolved a wide range of molecular and physiological mechanisms to buffer themselves from
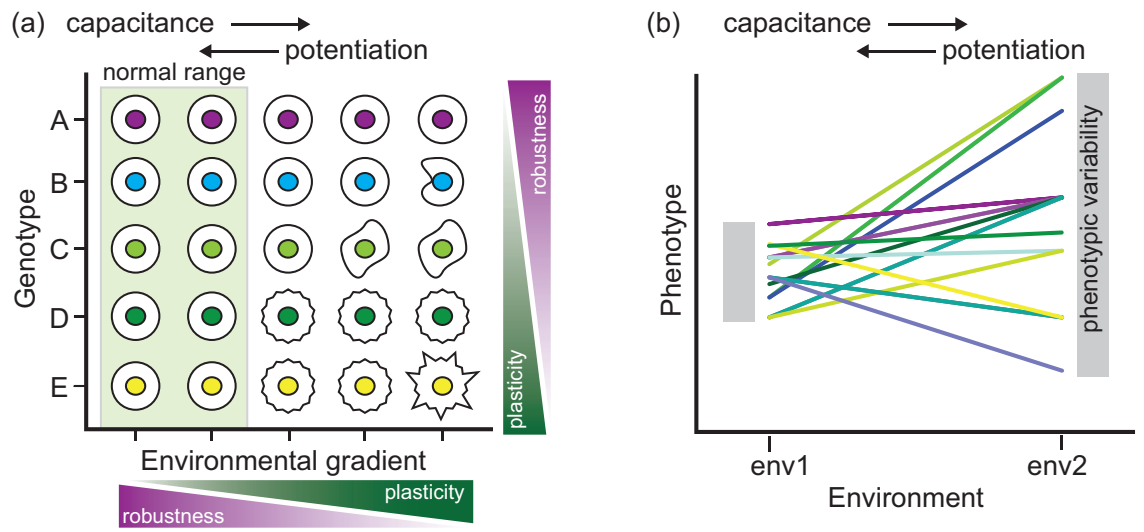

FIGURE 4.2 Robustness and plasticity in qualitative and quantitative traits. (a) Robustness and plasticity can be considered at the levels of a population or different individuals within that population. The $x$-axis demonstrates robustness and plasticity on a population scale. The phenotypic variability (number of external shapes) within the population increases from left to right. On the $y$-axis, individual genotypes show differential sensitivity (i.e., robustness or plasticity) to environmental perturbation. (b) The genotype-environment-phenotype relationships presented in Figure 1e are shown in a different form. Each colored line represents one of the genotypes in Figure 1e. A change from environment 1 to environment 2 results in phenotypic capacitance, an increase in heritable phenotypic variation. The reverse change would result in potentiation, a reduction in phenotypic variability. 
common environmental perturbations (de Visser et al. 2003; Felix and Wagner 2008; Levy and Siegal 2008; Masel and Siegal 2009; Frankel et al. 2010; Lempe et al. 2013; Siegal and Leu 2014). However, exposure to novel environments or highly stressful conditions may disrupt the robustness conferred by these buffering mechanisms. This can have consequences for the manifestation of heritable phenotypic variation because the molecular mechanisms that provide robustness to environmental perturbation may also buffer the effects of new mutations and standing genetic variants (Masel and Siegal 2009; Jarosz et al. 2010). When this occurs, over time a population may accumulate genetic variants with muted or 'cryptic' phenotypic effects in the ancestral or normal environmental range (Figure 4.2a) (Gibson and Dworkin 2004; Le Rouzic and Carlborg 2008; Paaby and Rockman 2014).

In a phenomenon referred to as 'phenotypic capacitance,' disruption of robustness by an environmental perturbation can increase the heritable phenotypic variation within a population by impairing the buffering of genetic variation (Rutherford and Lindquist 1998; Queitsch et al. 2002; Bergman and Siegal 2003; Jarosz and Lindquist 2010; Taylor and Ehrenreich 2015). Such environmental perturbations may also modify the effects of loci that were not previously buffered (Figure 4.2). In contrast to phenotypic capacitance, 'potentiation' can occur if an environmental perturbation causes genetic variants to lose their effects or exhibit reduced phenotypic impacts (Figure 4.2) (Jarosz and Lindquist 2010). These phenomena-phenotypic capacitance and potentiation - can substantially modify the relationship between genotype and phenotype, in turn impacting the levels and patterns of phenotypic diversity in a population (Figure 4.2) (Jarosz et al. 2010; Levy and Siegal 2012; Geiler-Samerotte et al. 2013; Schell et al. 2016).

To this juncture, we have discussed robustness as a generic feature of species and populations. However, genetically distinct individuals within a population can in fact vary in their levels of robustness (Queitsch et al. 2012; Felix and Barkoulas 2015; Ehrenreich and Pfennig 2016; Lee et al. 2016, 2019; Mestek Boukhibar and Barkoulas 2016). Differences in robustness among genotypes are caused by loci that individually and jointly render particular genotypes more or less sensitive to environmental change. Variation in robustness among genetically distinct individuals can have significant effects on the manifestation of plasticity across genotypes (Figure 4.2a). Importantly, through a phenomenon known as 'genetic accommodation,' natural selection can act on the genetic variation underlying plasticity to increase or decrease an organism's environmental responsiveness (West-Eberhard 2003). In some instances, this may result in 'genetic assimilation'-the transformation of an environmentally induced trait into a robust phenotype (Waddington 1953; see also Scheiner and Levis 2021 in this volume). Notably, the gain and subsequent loss of plasticity are thought to have a role in the emergence of phenotypic novelty (Baldwin 1902; Schmalhausen 1949; Waddington 1953; West-Eberhard 2003; see also Levis and Pfennig 2021; Pfennig 2021 in this volume), and such changes are likely mediated by loci influencing genotype-environment interaction, plasticity, and robustness.

As a concluding point, it bears mentioning that the concepts and terms presented here are often used differently depending upon the biological context and the perspective of the observer. Such confusion highlights the value of conceptualizing phenotypic plasticity as genotype-environment interaction; a perspective which is 
agnostic to whether or not heritable differences in plasticity fit the variable definitions of any given phenomenological case.

\subsection{METHODS FOR GENETICALLY DISSECTING PHENOTYPIC PLASTICITY}

A large body of theoretical research exists regarding the genetic basis of genotypeenvironment interaction and phenotypic plasticity (e.g., Via and Lande 1985; Scheiner 1993). In addition, there has also been a substantial effort to empirically address these topics. Often, such work requires 'mapping' the specific loci involved (Figure 4.3) (Fry et al. 1998; Ungerer et al. 2003; Juenger et al. 2005; Gutteling et al. 2007; El-Soda et al. 2014; Lowry et al. 2019), and ideally resolving these loci to the exact genes and genetic variants underlying them (Gerke et al. 2010; Lee et al. 2016, 2019; Matsui and Ehrenreich 2016). After causal loci have been comprehensively identified at high resolution, their phenotypic effects, as well as their interactions with each other and the environment, can be precisely measured (Gerke et al. 2010; Lee et al. 2016, 2019; Matsui and Ehrenreich 2016). These loci can also then be subjected to an additional study aimed at determining the mechanisms by which they affect plasticity.

The first steps in genetic mapping are 'genotyping' and 'phenotyping' (Mackay et al. 2009). Genotyping is the determination of the alleles that a number of genetically distinct individuals possess throughout their genomes. Phenotyping involves measuring traits of interest among genotyped individuals. For work focused on plasticity and genotype-environment interaction, traits must be measured across a number of distinct environments. Once genotypes and phenotypes have been obtained, statistical models can then be employed to identify contributing loci. Historically, the two main approaches for genetic mapping have been 'linkage mapping' and 'association mapping.' Linkage mapping relies on individuals from a known pedigree, which are usually progeny produced by crossing two or more genetically distinct individuals in a controlled manner (Figure 4.3a). In contrast, association mapping employs individuals that have been sampled from nature and have unknown pedigrees.

Linkage and association mapping each have advantages and disadvantages (Mackay et al. 2009). Because linkage mapping is limited to the genetic diversity present in cross parents, association mapping will generally capture more genetic diversity. Also, because natural populations experience many more meioses than occur during the construction of crosses, association mapping will often provide more precise resolution (e.g., Atwell et al. 2010; Brachi et al. 2010; Mackay et al. 2012). Yet, association mapping is confounded by non-causal correlations between genetic variants due to population structure (Pritchard et al. 2000; Zhao et al. 2007), whereas this is a more limited concern in linkage mapping. Furthermore, due to the balanced allele frequencies typically found in laboratory crosses, linkage mapping often affords a superior ability to detect loci (Ehrenreich et al. 2010; Bloom et al. 2013). This advantage is especially pronounced when it comes to detecting epistatic loci, which requires testing on allele combinations (Ehrenreich 2017). Often, the choice of approach will depend on features of the organism under study, such as generation time and amenability to controlled breeding. 
(a)

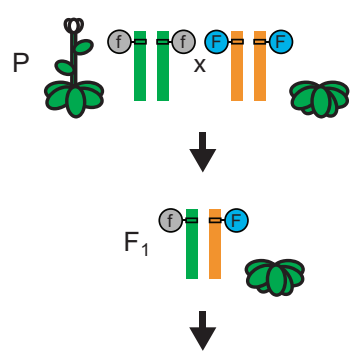

$\mathrm{F}_{2}$
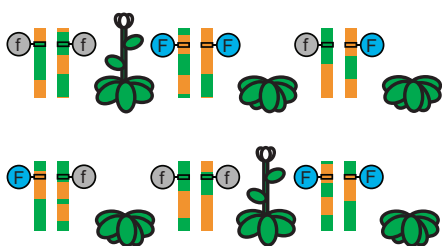

(b)

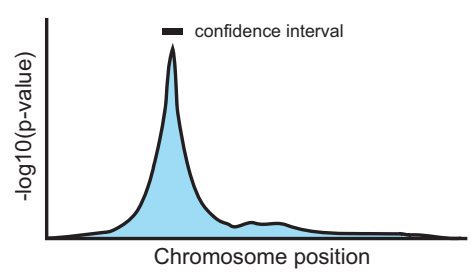

(c)

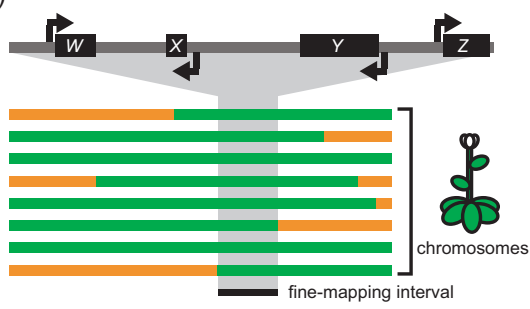

(d)

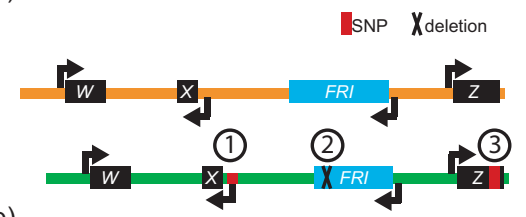

(e)
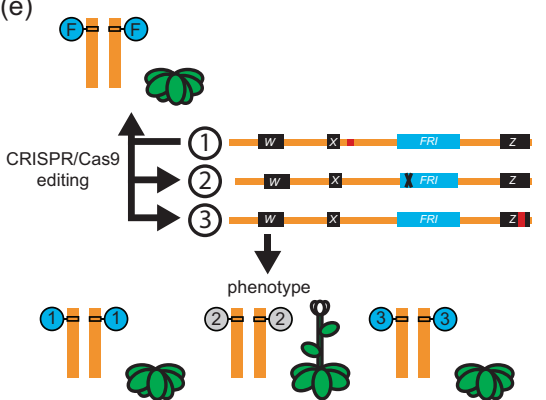

FIGURE 4.3 Mapping loci to the resolution of genes and genetic variants. (a) A typical experimental design for linkage mapping. Two genotyped strains are crossed to produce $F_{1}$ progeny, which are subsequently selfed or crossed to each other to produce an $F_{2}$ generation. The recombinant $\mathrm{F}_{2}$ progeny can then be phenotyped for traits of interest, in this case flowering in the absence of overwintering. Often, researchers will inbreed $\mathrm{F}_{2}$ strains to increase homozygosity and eliminate issues associated with allelic dominance. (b) A linkage scan identifies a highly significant locus on the examined chromosome. (c) Recombination breakpoints present among flowering offspring delimit the locus to four candidate genes. (d) Multiple candidate genetic variants are present in the delimited window of the chromosome. The two parents differ at two SNPs and a deletion. Note that this is an abstraction and real loci often harbor significantly more genetic variation. (e) Genome editing can be used to determine the exact causal genetic variant. Here, each of the candidate genetic variants $(1,2$, and 3$)$ is individually introduced into an otherwise isogenic genetic background using genome editing. 
Genetic mapping usually detects loci at the resolution of multiple genes and many genetic variants, limiting mechanistic inference (Figure 4.3b-d) (Mackay et al. 2009). Fortunately, there are strategies that can be employed to improve mapping resolution, such as using cross progeny from more than two parents (Kover et al. 2009; Aylor et al. 2011; King et al. 2012) or more than one generation of meiosis (Rockman and Kruglyak 2008; Linder et al. 2016), or by combining data from linkage and association mapping (Yu et al. 2008; Myles et al. 2009). Another option is to use 'site-directed mutagenesis,' a body of techniques for introducing targeted genetic changes into a genome, to resolve a locus spanning multiple genes down to a single gene (Figure 4.3d and e) (e.g., Steinmetz et al. 2002; Taylor and Ehrenreich 2014, 2015; Taylor et al. 2016). However, this approach often depends on the number of genes within a locus being small and the study organism allowing genetic manipulation.

Emerging tools for high-throughput site-directed and random mutagenesis are opening new doors for the genetic dissection of heritable traits, including environmentally responsive phenotypes. For example, improvements in CRISPR/Cas9mediated site-directed mutagenesis have made it possible to simultaneously explore the effects of thousands of single nucleotide changes in parallel (Roy et al. 2018; Sadhu et al. 2018; Sharon et al. 2018). Edited pools of cells can be grown in different environments to identify exact nucleotide variants that interact with the environment. Another approach with similar potential is 'reciprocal hemizygosity analysis,' a technique that utilizes gene disruption to enable comparison of individuals that carry only one copy of a gene of interest in an otherwise isogenic, diploid genome (Steinmetz et al. 2002). Recently, it was shown that transposon mutagenesis can be used to perform reciprocal hemizygosity analysis on a genome-wide scale (Weiss et al. 2018). Presently, these technologies work best in organisms that are predominantly unicellular, such as the budding yeast Saccharomyces cerevisiae, or in cell lines derived from particular tissues.

\subsection{EMPIRICAL INSIGHTS INTO THE GENETICS OF PHENOTYPIC PLASTICITY}

A number of high resolution studies of natural populations have characterized how segregating loci contribute to phenotypic plasticity and genotype-environment interaction (e.g., Aukerman et al. 1997; Johanson et al. 2000; El-Din El-Assal et al. 2001; Maloof et al. 2001; Werner et al. 2005; Balasubramanian et al. 2006; Filiault et al. 2008; Gerke et al. 2010; Lee et al. 2016, 2019; Matsui and Ehrenreich 2016). Such studies have mainly been conducted in model organisms, such as the thale cress, Arabidopsis thaliana and budding yeast, S. cerevisiae. These, and other, model organisms possess features that facilitate studying genotype-environment interaction, and are accompanied by deep knowledge bases and powerful toolkits for molecular genetics and functional genomics (Koornneef et al. 2004; Shindo et al. 2007; Ehrenreich et al. 2009a; Liti and Louis 2012; Weigel 2012; Ehrenreich and Magwene 2017). Critically, these foundations make it possible to not only identify causal genes and genetic variants but also situate newly identified genetic factors within biological networks. 
Arabidopsis thaliana, the main plant model system, offers numerous advantages for studying the genetic basis of plasticity. This species is predominantly self-fertilizing; thus, strains isolated from nature are typically highly inbred and homozygous throughout their genomes (Koornneef et al. 2004; Shindo et al. 2007; Weigel 2012). Because these strains are genetically stable, they can be grown in parallel in a number of distinct environments, facilitating the study of plasticity using association mapping (Ehrenreich et al. 2009b; Atwell et al. 2010). Linkage disequilibrium decays rapidly in A. thaliana, meaning that loci are often detected at high resolution by association mapping (Nordborg et al. 2005; Weigel and Nordborg 2005). In addition, $A$. thaliana has retained the ability to outcross, and strains can easily be mated to each other to facilitate linkage mapping (Ungerer et al. 2003; Juenger et al. 2005). Furthermore, because A. thaliana is highly amenable to genetic engineering (Miki et al. 2018), detected loci can also be resolved and validated using mutagenesis techniques.

Linkage and association studies in A. thaliana have found an abundance of environmentally responsive genetic variation in nearly all traits, including flowering time (Aukerman et al. 1997; Johanson et al. 2000; El-Din El-Assal et al. 2001; Maloof et al. 2001; Werner et al. 2005; Balasubramanian et al. 2006; Filiault et al. 2008), root architecture (Rosas et al. 2013), pathogen response (Corwin and Kliebenstein 2017), and nutrient utilization (Baxter et al. 2012). Flowering time, in particular, has served as a model phenotype for understanding the genetic architecture of plasticity (Mouradov et al. 2002). Evidence suggests plasticity in flowering time in A. thaliana (Zhao et al. 2007; Ehrenreich et al. 2009b; Kover et al. 2009; Atwell et al. 2010; Salome et al. 2011), as well as in other plant species (Gage et al. 2017; Lowry et al. 2019), involves a large number of distinct loci.

Many genes and genetic variants that contribute to heritable variation in flowering time across environments have been identified in A. thaliana (Aukerman et al. 1997; El-Din El-Assal et al. 2001; Maloof et al. 2001; Werner et al. 2005; Balasubramanian et al. 2006; Filiault et al. 2008; Ehrenreich et al. 2009b; Atwell et al. 2010). In many cases, these genes are the same genes identified in classic genetic screens focused on flowering time. However, in some instances, the study of plasticity and genotypeenvironment interaction has led to the discovery of novel, environmentally responsive genes. Arguably, the best example of this is FRIGIDA (FRI), which encodes a component of a regulatory complex that helps suppress flowering until after winter (Figure 4.4a) (Johanson et al. 2000). Many strains carry non-functional FRI alleles, meaning they can flower without experiencing overwintering (or vernalization), resulting in a striking example of gene-environment interaction (Figure 4.4a) (Gazzani et al. 2003).

Although A. thaliana has provided valuable insights into mechanisms underlying plasticity and genotype-environment interaction in natural environments, this system is more limited in its ability to shed light on the role of genetic interactions. In this regard, S. cerevisiae has provided insights that would have been difficult to obtain using other systems (Ehrenreich et al. 2009a; Liti and Louis 2012; Ehrenreich and Magwene 2017; Yadav and Sinha 2018). Yeast grows rapidly under controlled laboratory conditions that are easily manipulated. Additionally, it is possible to culture hundreds, if not thousands, of genetically distinct yeast strains at the same time. 
(a)
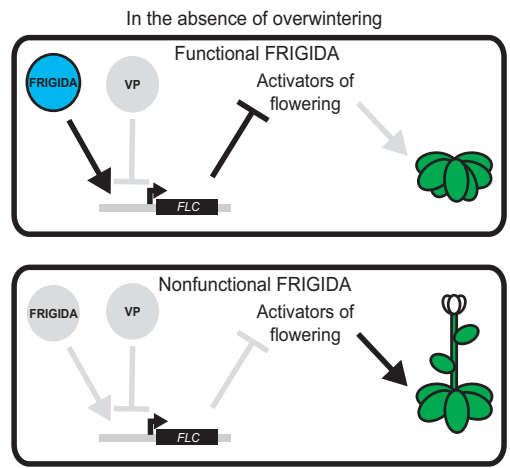

In the presence of overwintering

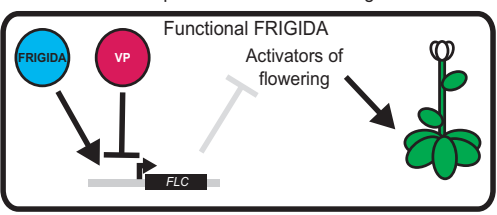

(b)

genotypes

phenotypes

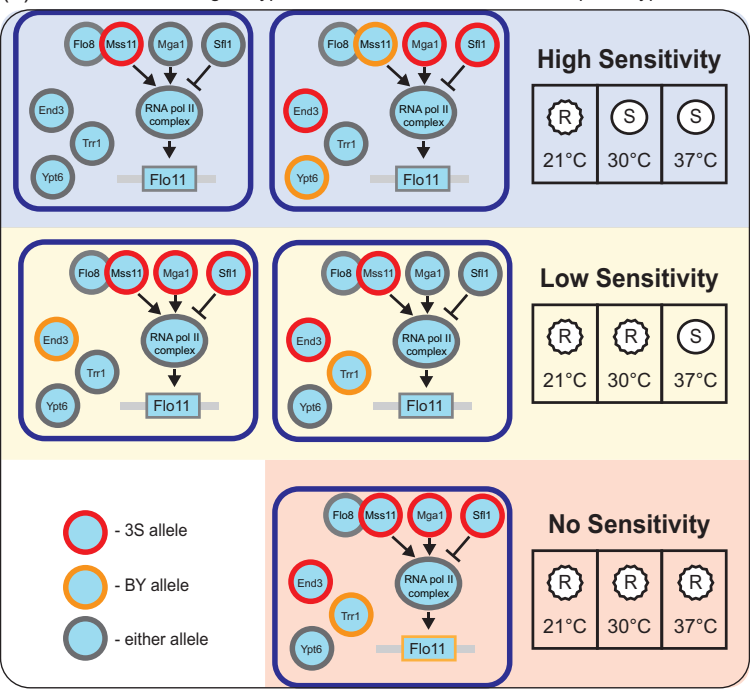

FIGURE 4.4 Genetic mechanisms underlying phenotypic plasticity. (a) A simplified mechanism for plasticity in flowering in Arabidopsis. A functional FRIGIDA (FRI) gene in the absence of overwintering induces the expression of FLOWERING LOCUS C (FLC), which is a strong repressor of flowering. Without a functional FRI, FLC is not expressed and flowering proceeds. Overwintering induces the expression of genes in the vernalization pathway. These environmentally sensitive genes counteract $F R I$ by inducing repressive chromatin at the FLC promoter. Vernalization enables plants to flower by nullifying the effect of FRI. (b) Complex genetic architectures of an environmentally responsive rough colony phenotype in a cross of the $S$. cerevisiae strains BY and 3S. Linkage mapping in a yeast cross identified five distinct genotypes composed of seven environmentally sensitive loci that produce rough colonies with varying levels of temperature sensitivity. Most of the involved genes play roles in the same pathway, which regulates the transcription of FLO11. 
Although association mapping is of limited utility in yeast due to its extensive population structure (Peter et al. 2018), easy cross generation, genotyping, and phenotyping enable statistically powerful genetic mapping via linkage studies (Ehrenreich et al. 2010; Bloom et al. 2013). Thus, with yeast, the genetic architectures of plasticity, including epistatic effects, between a small number of strains can be determined to near completion.

In yeast, evidence supports a model in which environmentally responsive genetic variants in different genes genetically interact to impact the activity of particular pathways that control the transcription of master regulators of cell fate. At least two examples supporting such a model have been comprehensively dissected: one in sporulation (Gerke et al. 2009, 2010) and the other in colony morphology (Figure 4.4b) (Taylor and Ehrenreich 2014, 2015; Lee et al. 2016, 2019; Taylor et al. 2016). In both examples, involved genetic variants interacted with each other and the environment to regulate the transcription of a key master regulator-IME1 and FLOII in the cases of sporulation and colony morphology, respectively. Interestingly, in the case of colony morphology, environmentally responsive genetic interactions modulated sensitivity of the trait to temperature, with some genotypes even showing robustness to temperature (Figure 4.4b) (Lee et al. 2016, 2019).

However, plasticity need not arise through highly epistatic genetic architectures mediated by a single master regulator. Genotype-environment interactions can also involve large numbers of environmentally sensitive loci that exhibit additive effects (Matsui and Ehrenreich 2016; Wei and Zhang 2017) or a mixture of additive and epistatic effects (Bhatia et al. 2014; Yadav et al. 2016). For example, one study in yeast found that genetic variants in unrelated components of the stress response machinery showed additive effects on genotype-environment interaction (Matsui and Ehrenreich 2016). Undoubtedly, other studies that achieve comparable resolution will provide additional examples of distinct molecular mechanisms.

Collectively, studies like the ones described here suggest a number of features regarding the genetic basis of variation in plasticity in natural populations. Plasticity can be controlled by many loci that may interact with not only the environment but also each other. Genetic variants that impact the transcription of key genes may be a common, although not necessarily universal, source of gene- and genotypeenvironment interaction. This makes sense given that most species harbor an abundance of gene- and genotype-environment interaction in gene regulation (Smith and Kruglyak 2008; Maranville et al. 2012; Grishkevich and Yanai 2013). Yet, it is also clear that there is no singular genetic architecture or mechanism that entirely explains plasticity and genotype-environment interaction in populations. These insights indicate that the genetic basis of plasticity will likely depend on the species, traits, and environments under study.

\subsection{THE FUTURE OF THE GENETICS OF PHENOTYPIC PLASTICITY}

To this point, we have discussed how genetic variation can interact with the environment to alter phenotype. We have also described how these genotype-environment interactions, visible at the phenotypic level, result from genetic variants altering the molecular function of genes in response to the environment. In this section, we 
propose some next steps that have the potential to transform our understanding of the genetic basis of plasticity.

First, non-model species should increasingly be used as focal systems for studying the genetic basis of plasticity. Although the majority of high-resolution research on genetic variation in plasticity has occurred in model systems with well-developed molecular genetics and functional genomics toolkits, important examples exist where researchers have studied the genetic basis of plasticity in non-model organisms (e.g., Sieriebriennikov et al. 2018). Technological advances are reducing the barriers to genetic and genomic research in non-model species and should make it easier to study genetic variation in plasticity in non-model species. For example, progress in sequencing technologies and genome assembly have now made it possible to produce high quality, telomere-to-telomere genomes for almost any organism, as discussed in (Ekblom and Wolf 2014; Simpson and Pop 2015; Jung et al. 2019) and elsewhere. In addition, CRISPR/Cas systems should enable genetic manipulation in nearly any species (Cong et al. 2013; Mali et al. 2013). These technological advances will increasingly facilitate studying the genetic basis of plasticity in non-model species that possess compelling environmentally responsive phenotypes (e.g., Ragsdale and Ivers 2016).

Second, we need a better understanding of how genetic variants that interact with the environment impact entire networks of genes and proteins. Here, it is important to recognize that a genetic variant affecting one gene is likely, in turn, to have downstream consequences for the functions of many other genes. Understanding these downstream consequences becomes especially important when one considers the potential for natural selection to act on and modify plasticity. For example, whether genetic assimilation can occur depends on a system allowing for, and tolerating, genetic variants that promote robustness. Accessing the systems biology of plasticity will likely require combining the genetic mapping approaches described earlier with transcriptomic, proteomic, and other high-dimensionality datasets produced from the same individuals in multiple environments (Sieberts and Schadt 2007; Ayroles et al. 2009; Nadeau and Dudley 2011; Civelek and Lusis 2014).

Third, for multicellular organisms in particular, insight is needed into how genotype-environment interactions impact development. New technologies have made it possible to approach this difficult problem. For example, high-throughput single-cell RNA sequencing enables the generation of transcriptomes from thousands of distinct cells from a given tissue (Macosko et al. 2015; Satija et al. 2015; Briggs et al. 2018; Farrell et al. 2018; Wagner et al. 2018). In addition, CRISPR/ Cas9-mediated developmental barcoding provides ontogenetic histories of organs, tissues, and even individual cells (McKenna et al. 2016; Alemany et al. 2018; Kalhor et al. 2018). It may be possible to use these approaches individually or in combination (Kester and van Oudenaarden 2018; Raj et al. 2018; Baron and van Oudenaarden 2019; Wagner and Klein 2020) to learn how genotype-environment interactions modify the developmental process itself. Such research might also provide insights into the relationship between heritable differences in phenotypic plasticity and epigenetic contributions to plasticity.

These ideas illustrate how the next step in understanding the genetics of plasticity is to move beyond standard genetics in model organisms and to begin to better 
determine the mechanisms by which genetic variation modifies the relationship between genotype, environment, and phenotype across a broader array of species. Doing this will require characterizing how genetic variants impact the networks underpinning environmentally responsive phenotypes and examining how plasticity manifests during development.

\subsection{CONCLUSIONS}

Genetically distinct individuals commonly exhibit heritable differences in their phenotypic responses to the environment. This variation in plasticity is a direct result of loci that harbor genetic variants that individually show gene-environment interactions and jointly give rise to genotype-environment interactions. These genetic variants impact the relationship between genotype, environment, and phenotype by modifying how genes function at the molecular level in response to the environment. Because many such loci often segregate within populations, environmental changes can significantly alter the phenotypic variation exhibited by a population, thereby providing a potential substrate for evolutionary change.

Understanding how genetic variation gives rise to these differences in plasticity is important within the contexts of genetics and evolutionary biology. Indeed, such research can shed light on the mechanisms underlying genetic accommodation and genetic assimilation, which, in turn, has the potential to improve knowledge of the environment's role in facilitating the evolution of novel traits. Because genetic variation in plasticity may also impact how populations and species respond to global climate change, research on this topic may also be generally informative regarding the future evolutionary trajectories of life on our planet. Additionally, it is important to recognize that genetic variation in plasticity has bearing on all other areas of biology in which the relationship between genotype, environment, and phenotype matters, including agriculture, medicine, and synthetic biology.

In this paper, we have broadly discussed heritable variation in plasticity from both conceptual and empirical perspectives, emphasizing what is known and what still needs to be learned. Central to this discussion is the importance of understanding mechanism: How do environmentally responsive genetic variants individually and jointly impact the functions of individual genes? How do they affect the activities of pathways, complexes, and networks? And, how do they impact developmental processes? Answering these questions will produce valuable, detailed, descriptive insights into plasticity that can inform all areas of biology and ideally facilitate a future in which our knowledge of plasticity enables the prediction of how organisms will respond to the environment based on their genotypes. We provide some suggestions for future research in Box 4.1.

\section{ACKNOWLEDGMENTS}

We thank members of the Ehrenreich lab, Andrew Isdaner, David Pfennig, and two anonymous reviewers for their input on a draft of this manuscript. This work was supported by grant R35GM130381 from the National Institutes of Health to I.M.E., as well as funds from the University of Southern California to I.M.E. 


\section{BOX 4.1 SUGGESTIONS FOR FUTURE RESEARCH}

- What are the genetic architectures and molecular mechanisms underlying phenotypic plasticity across a broader range of species? Such work can determine the generality of findings to date, such as the observation of high genetic complexity. Application of this work to a diversity of continuous and discrete traits that have experienced varying degrees of natural selection could connect empirical findings about genetic architecture to ecological and evolutionary theory.

- What is the nature of the gene- and genotype-environment interactions that underlie variation in plasticity? It is unclear to what extent environmentally induced changes in heritable phenotypic variation are mediated by cryptic genetic variation as opposed to genetic variation that is already visible. Further work is needed to not only identify at high-resolution loci that contribute to plasticity, but also examine how the effects of these loci change and genetically interact with each other across environments.

- Can we obtain a clearer understanding of molecular, cellular, and developmental mechanisms underlying genetic differences in plasticity? This involves developing a more general understanding of how individual genetic variants contribute to plastic phenotypes across environments. However, it is also necessary to assess how these variants combine to impact the function of pathways and networks. These consequences should be examined within and across cells, tissues, and sexes. The latter work can potentially shed light on how geneand genotype-environment interactions modify developmental processes in multicellular organisms.

- What are the genetic and molecular bases of genetic accommodation and assimilation? A major reason why genetic variation in plasticity is interesting is because it can potentially help us understand how evolution works. Gene- and genotype-environment interactions contributing to variation in plasticity provide a crucial substrate for selection to act upon to modify the relationship between genotype, environment, and phenotype. Thus, it may be that empirically studying genetic variation in plasticity can provide valuable insights into the mechanisms of genetic accommodation and assimilation.

\section{REFERENCES}

Alemany, A., M. Florescu, C. S. Baron, J. Peterson-Maduro, and A. van Oudenaarden. 2018. Whole-organism clone tracing using single-cell sequencing. Nature 556:108-112.

Atwell, S., Y. S. Huang, B. J. Vilhjalmsson, G. Willems, M. Horton, Y. Li, D. Meng, A. Platt, A. M. Tarone, T. T. Hu, R. Jiang, N. W. Muliyati, X. Zhang, M. A. Amer, I. Baxter, B. Brachi, J. Chory, C. Dean, M. Debieu, J. de Meaux, J. R. Ecker, N. Faure, J. M. Kniskern, J. D. Jones, T. Michael, A. Nemri, F. Roux, D. E. Salt, C. Tang, M. Todesco, 
M. B. Traw, D. Weigel, P. Marjoram, J. O. Borevitz, J. Bergelson, and M. Nordborg. 2010. Genome-wide association study of 107 phenotypes in Arabidopsis thaliana inbred lines. Nature 465:627-631.

Aubin-Horth, N. and S. C. Renn. 2009. Genomic reaction norms: Using integrative biology to understand molecular mechanisms of phenotypic plasticity. Mol Ecol 18:3763-3780.

Aukerman, M. J., M. Hirschfeld, L. Wester, M. Weaver, T. Clack, R. M. Amasino, and R. A. Sharrock. 1997. A deletion in the PHYD gene of the Arabidopsis Wassilewskija ecotype defines a role for phytochrome D in red/far-red light sensing. Plant Cell 9:1317-1326.

Aylor, D. L., W. Valdar, W. Foulds-Mathes, R. J. Buus, R. A. Verdugo, R. S. Baric, M. T. Ferris, J. A. Frelinger, M. Heise, M. B. Frieman, L. E. Gralinski, T. A. Bell, J. D. Didion, K. Hua, D. L. Nehrenberg, C. L. Powell, J. Steigerwalt, Y. Xie, S. N. Kelada, F. S. Collins, I. V. Yang, D. A. Schwartz, L. A. Branstetter, E. J. Chesler, D. R. Miller, J. Spence, E. Y. Liu, L. McMillan, A. Sarkar, J. Wang, W. Wang, Q. Zhang, K. W. Broman, R. Korstanje, C. Durrant, R. Mott, F. A. Iraqi, D. Pomp, D. Threadgill, F. P. de Villena, and G. A. Churchill. 2011. Genetic analysis of complex traits in the emerging Collaborative Cross. Genome Res 21:1213-1222.

Ayroles, J. F., M. A. Carbone, E. A. Stone, K. W. Jordan, R. F. Lyman, M. M. Magwire, S. M. Rollmann, L. H. Duncan, F. Lawrence, R. R. Anholt, and T. F. Mackay. 2009. Systems genetics of complex traits in Drosophila melanogaster. Nat Genet 41:299-307.

Balasubramanian, S., S. Sureshkumar, M. Agrawal, T. P. Michael, C. Wessinger, J. N. Maloof, R. Clark, N. Warthmann, J. Chory, and D. Weigel. 2006. The PHYTOCHROME C photoreceptor gene mediates natural variation in flowering and growth responses of Arabidopsis thaliana. Nat Genet 38:711-715.

Baldwin, J. M. 1902. Development and Evolution. Macmillan, New York.

Baron, C. S. and A. van Oudenaarden. 2019. Unravelling cellular relationships during development and regeneration using genetic lineage tracing. Nat Rev Mol Cell Biol 20:753-765.

Bateson, P. and P. Gluckman. 2011. Plasticity, Robustness, Development and Evolution. Cambridge University Press, Cambride, UK.

Baxter, I., C. Hermans, B. Lahner, E. Yakubova, M. Tikhonova, N. Verbruggen, D. Y. Chao, and D. E. Salt. 2012. Biodiversity of mineral nutrient and trace element accumulation in Arabidopsis thaliana. PLoS One 7:e35121.

Baye, T. M., T. Abebe, and R. A. Wilke. 2011. Genotype-environment interactions and their translational implications. Per Med 8:59-70.

Beldade, P., A. R. Mateus, and R. A. Keller. 2011. Evolution and molecular mechanisms of adaptive developmental plasticity. Mol Ecol 20:1347-1363.

Bergman, A. and M. L. Siegal. 2003. Evolutionary capacitance as a general feature of complex gene networks. Nature 424:549-552.

Bhatia, A., A. Yadav, C. Zhu, J. Gagneur, A. Radhakrishnan, L. M. Steinmetz, G. Bhanot, and H. Sinha. 2014. Yeast growth plasticity is regulated by environment-specific multi-QTL interactions. G3 (Bethesda) 4:769-777.

Bloom, J. S., I. M. Ehrenreich, W. T. Loo, T. L. Lite, and L. Kruglyak. 2013. Finding the sources of missing heritability in a yeast cross. Nature 494:234-237.

Brachi, B., N. Faure, M. Horton, E. Flahauw, A. Vazquez, M. Nordborg, J. Bergelson, J. Cuguen, and F. Roux. 2010. Linkage and association mapping of Arabidopsis thaliana flowering time in nature. PLoS Genet 6:e1000940.

Bradshaw, A. D. 1965. Evolutionary significance of phenotypic plasticity in plants. Adv Genet 13:115-155.

Briggs, J. A., C. Weinreb, D. E. Wagner, S. Megason, L. Peshkin, M. W. Kirschner, and A. M. Klein. 2018. The dynamics of gene expression in vertebrate embryogenesis at singlecell resolution. Science 360:eaar5780.

Civelek, M. and A. J. Lusis. 2014. Systems genetics approaches to understand complex traits. Nat Rev Genet 15:34-48. 
Cong, L., F. A. Ran, D. Cox, S. Lin, R. Barretto, N. Habib, P. D. Hsu, X. Wu, W. Jiang, L. A. Marraffini, and F. Zhang. 2013. Multiplex genome engineering using CRISPR/Cas systems. Science 339:819-823.

Corwin, J. A. and D. J. Kliebenstein. 2017. Quantitative resistance: More than just perception of a pathogen. Plant Cell 29:655-665.

de Visser, J. A., J. Hermisson, G. P. Wagner, L. Ancel Meyers, H. Bagheri-Chaichian, J. L. Blanchard, L. Chao, J. M. Cheverud, S. F. Elena, W. Fontana, G. Gibson, T. F. Hansen, D. Krakauer, R. C. Lewontin, C. Ofria, S. H. Rice, G. von Dassow, A. Wagner, and M. C. Whitlock. 2003. Perspective: Evolution and detection of genetic robustness. Evolution 57:1959-1972.

Debat, V. and P. David. 2001. Mapping phenotypes: Canalization, plasticity and developmental stability. Trends Ecol Evol 16:555-561.

Dufty Jr, A. M., J. Clobert, and A. P. Møller. 2002. Hormones, developmental plasticity and adaptation. Trends Ecol Evol 17:190-196.

Ehrenreich, I. M. 2017. Epistasis: Searching for interacting genetic variants using crosses. Genetics 206:531-535.

Ehrenreich, I. M., J. P. Gerke, and L. Kruglyak. 2009a. Genetic dissection of complex traits in yeast: Insights from studies of gene expression and other phenotypes in the BYxRM cross. Cold Spring Harb Symp Quant Biol 74:145-153.

Ehrenreich, I. M., Y. Hanzawa, L. Chou, J. L. Roe, P. X. Kover, and M. D. Purugganan. 2009b. Candidate gene association mapping of Arabidopsis flowering time. Genetics 183:325-335.

Ehrenreich, I. M. and P. M. Magwene. 2017. Genetic analysis of complex traits in Saccharomyces cerevisiae. Cold Spring Harb Protoc. doi:10.1101/pdb.top077602.

Ehrenreich, I. M. and D. W. Pfennig. 2016. Genetic assimilation: A review of its potential proximate causes and evolutionary consequences. Ann Bot 117:769-779.

Ehrenreich, I. M., N. Torabi, Y. Jia, J. Kent, S. Martis, J. A. Shapiro, D. Gresham, A. A. Caudy, and L. Kruglyak. 2010. Dissection of genetically complex traits with extremely large pools of yeast segregants. Nature 464:1039-1042.

Ekblom, R. and J. B. Wolf. 2014. A field guide to whole-genome sequencing, assembly and annotation. Evol Appl 7:1026-1042.

El-Din El-Assal, S., C. Alonso-Blanco, A. J. Peeters, V. Raz, and M. Koornneef. 2001. A QTL for flowering time in Arabidopsis reveals a novel allele of CRY2. Nat Genet 29:435-440.

El-Soda, M., M. Malosetti, B. J. Zwaan, M. Koornneef, and M. G. Aarts. 2014. Genotypexenvironment interaction QTL mapping in plants: Lessons from Arabidopsis. Trends Plant Sci 19:390-398.

Falconer, D. S. and T. F. C. Mackay. 1996. Introduction to Quantitative Genetics. Longmans Green, Essex, UK.

Farrell, J. A., Y. Wang, S. J. Riesenfeld, K. Shekhar, A. Regev, and A. F. Schier. 2018. Singlecell reconstruction of developmental trajectories during zebrafish embryogenesis. Science 360: eaar3131.

Felix, M. A. and M. Barkoulas. 2015. Pervasive robustness in biological systems. Nat Rev Genet 16:483-496.

Felix, M. A. and A. Wagner. 2008. Robustness and evolution: Concepts, insights and challenges from a developmental model system. Heredity (Edinb) 100:132-140.

Filiault, D. L., C. A. Wessinger, J. R. Dinneny, J. Lutes, J. O. Borevitz, D. Weigel, J. Chory, and J. N. Maloof. 2008. Amino acid polymorphisms in Arabidopsis phytochrome B cause differential responses to light. Proc Natl Acad Sci USA 105:3157-3162.

Flint, J. and T. F. Mackay. 2009. Genetic architecture of quantitative traits in mice, flies, and humans. Genome Res 19:723-733.

Frankel, N., G. K. Davis, D. Vargas, S. Wang, F. Payre, and D. L. Stern. 2010. Phenotypic robustness conferred by apparently redundant transcriptional enhancers. Nature 466:490-493. 
Fry, J. D., S. V. Nuzhdin, E. G. Pasyukova, and T. F. Mackay. 1998. QTL mapping of genotypeenvironment interaction for fitness in Drosophila melanogaster. Genet Res 71:133-141.

Gage, J. L., D. Jarquin, C. Romay, A. Lorenz, E. S. Buckler, S. Kaeppler, N. Alkhalifah, M. Bohn, D. A. Campbell, J. Edwards, D. Ertl, S. Flint-Garcia, J. Gardiner, B. Good, C. N. Hirsch, J. Holland, D. C. Hooker, J. Knoll, J. Kolkman, G. Kruger, N. Lauter, C. J. Lawrence-Dill, E. Lee, J. Lynch, S. C. Murray, R. Nelson, J. Petzoldt, T. Rocheford, J. Schnable, P. S. Schnable, B. Scully, M. Smith, N. M. Springer, S. Srinivasan, R. Walton, T. Weldekidan, R. J. Wisser, W. Xu, J. Yu, and N. de Leon. 2017. The effect of artificial selection on phenotypic plasticity in maize. Nat Commun 8:1348.

Gazzani, S., A. R. Gendall, C. Lister, and C. Dean. 2003. Analysis of the molecular basis of flowering time variation in Arabidopsis accessions. Plant Physiol 132:1107-1114.

Geiler-Samerotte, K. A., C. R. Bauer, S. Li, N. Ziv, D. Gresham, and M. L. Siegal. 2013. The details in the distributions: Why and how to study phenotypic variability. Curr Opin Biotechnol 24:752-759.

Gerke, J., K. Lorenz, and B. Cohen. 2009. Genetic interactions between transcription factors cause natural variation in yeast. Science 323:498-501.

Gerke, J., K. Lorenz, S. Ramnarine, and B. Cohen. 2010. Gene-environment interactions at nucleotide resolution. PLoS Genet 6:e1001144.

Gibson, G. and I. Dworkin. 2004. Uncovering cryptic genetic variation. Nat Rev Genet 5:681-690.

Grishkevich, V. and I. Yanai. 2013. The genomic determinants of genotype x environment interactions in gene expression. Trends Genet 29:479-487.

Gutteling, E. W., J. A. Riksen, J. Bakker, and J. E. Kammenga. 2007. Mapping phenotypic plasticity and genotype-environment interactions affecting life-history traits in Caenorhabditis elegans. Heredity (Edinb) 98:28-37.

Hansen, T. F. 2006. The evolution of genetic architecture. Annu Rev Ecol Evol Syst 37:123-157.

Hunter, D. J. 2005. Gene-environment interactions in human diseases. Nat Rev Genet 6:287-298.

Jarosz, D. F. and S. Lindquist. 2010. Hsp90 and environmental stress transform the adaptive value of natural genetic variation. Science 330:1820-1824.

Jarosz, D. F., M. Taipale, and S. Lindquist. 2010. Protein homeostasis and the phenotypic manifestation of genetic diversity: Principles and mechanisms. Annu Rev Genet 44:189-216.

Johanson, U., J. West, C. Lister, S. Michaels, R. Amasino, and C. Dean. 2000. Molecular analysis of FRIGIDA, a major determinant of natural variation in Arabidopsis flowering time. Science 290:344-347.

Juenger, T. E., S. Sen, K. A. Stowe, and E. L. Simms. 2005. Epistasis and genotype-environment interaction for quantitative trait loci affecting flowering time in Arabidopsis thaliana. Genetica 123:87-105.

Jung, H., C. Winefield, A. Bombarely, P. Prentis, and P. Waterhouse. 2019. Tools and strategies for long-read sequencing and de novo assembly of plant genomes. Trends Plant Sci 24:700-724.

Kalhor, R., K. Kalhor, L. Mejia, K. Leeper, A. Graveline, P. Mali, and G. M. Church. 2018. Developmental barcoding of whole mouse via homing CRISPR. Science 361:eaat9804.

Kang, M. S. 1997. Using genotype-by-environment interaction for crop cultivar development. Adv Agron 62:199-252.

Kester, L. and A. van Oudenaarden. 2018. Single-cell transcriptomics meets lineage tracing. Cell Stem Cell 23:166-179.

King, E. G., S. J. Macdonald, and A. D. Long. 2012. Properties and power of the Drosophila synthetic population resource for the routine dissection of complex traits. Genetics 191:935-949.

Kitano, H. 2004. Biological robustness. Nat Rev Genet 5:826-837.

Koornneef, M., C. Alonso-Blanco, and D. Vreugdenhil. 2004. Naturally occurring genetic variation in Arabidopsis thaliana. Annu Rev Plant Biol 55:141-172. 
Kover, P. X., W. Valdar, J. Trakalo, N. Scarcelli, I. M. Ehrenreich, M. D. Purugganan, C. Durrant, and R. Mott. 2009. A multiparent advanced generation inter-cross to fine-map quantitative traits in Arabidopsis thaliana. PLoS Genet 5:e1000551.

Lafuente, E. and P. Beldade. 2019. Genomics of developmental plasticity in animals. Front Genet 10:720.

Le Rouzic, A. and O. Carlborg. 2008. Evolutionary potential of hidden genetic variation. Trends Ecol Evol 23:33-37.

Lee, J. T., A. L. V. Coradini, A. Shen, and I. M. Ehrenreich. 2019. Layers of cryptic genetic variation underlie a yeast complex trait. Genetics 211:1469-1482.

Lee, J. T., M. B. Taylor, A. Shen, and I. M. Ehrenreich. 2016. Multi-locus genotypes underlying temperature sensitivity in a mutationally induced trait. PLoS Genet 12:e1005929.

Lempe, J., J. Lachowiec, A. M. Sullivan, and C. Queitsch. 2013. Molecular mechanisms of robustness in plants. Curr Opin Plant Biol 16:62-69.

Levis, N. A. and D. W. Pfennig. 2021. Innovation and diversification via plasticity-led evolution. In D. W. Pfennig, ed., Phenotypic Plasticity and Evolution: Causes, Consequences, Controversies. CRC Press, Boca Raton, FL.

Levy, S. F. and M. L. Siegal. 2008. Network hubs buffer environmental variation in Saccharomyces cerevisiae. PLoS Biol 6:e264.

Levy, S. F. and M. L. Siegal. 2012. The robustness continuum. Adv Exp Med Biol 751:431-452.

Linder, R. A., F. Seidl, K. Ha, and I. M. Ehrenreich. 2016. The complex genetic and molecular basis of a model quantitative trait. Mol Biol Cell 27:209-218.

Liti, G. and E. J. Louis. 2012. Advances in quantitative trait analysis in yeast. PLoS Genet 8:e1002912.

Lowry, D. B., J. T. Lovell, L. Zhang, J. Bonnette, P. A. Fay, R. B. Mitchell, J. Lloyd-Reilley, A. R. Boe, Y. Wu, F. M. Rouquette, Jr., R. L. Wynia, X. Weng, K. D. Behrman, A. Healey, K. Barry, A. Lipzen, D. Bauer, A. Sharma, J. Jenkins, J. Schmutz, F. B. Fritschi, and T. E. Juenger. 2019. QTL $x$ environment interactions underlie adaptive divergence in switchgrass across a large latitudinal gradient. Proc Natl Acad Sci USA 116:12933-12941.

Lynch, M. and B. Walsh. 1998. Genetics and Analysis of Quantitative Traits. Sinauer, Sunderland, MA.

Mackay, T. F. 2001. The genetic architecture of quantitative traits. Annu Rev Genet 35:303-339.

Mackay, T. F. 2014. Epistasis and quantitative traits: Using model organisms to study genegene interactions. Nat Rev Genet 15:22-33.

Mackay, T. F., S. Richards, E. A. Stone, A. Barbadilla, J. F. Ayroles, D. Zhu, S. Casillas, Y. Han, M. M. Magwire, J. M. Cridland, M. F. Richardson, R. R. Anholt, M. Barron, C. Bess, K. P. Blankenburg, M. A. Carbone, D. Castellano, L. Chaboub, L. Duncan, Z. Harris, M. Javaid, J. C. Jayaseelan, S. N. Jhangiani, K. W. Jordan, F. Lara, F. Lawrence, S. L. Lee, P. Librado, R. S. Linheiro, R. F. Lyman, A. J. Mackey, M. Munidasa, D. M. Muzny, L. Nazareth, I. Newsham, L. Perales, L. L. Pu, C. Qu, M. Ramia, J. G. Reid, S. M. Rollmann, J. Rozas, N. Saada, L. Turlapati, K. C. Worley, Y. Q. Wu, A. Yamamoto, Y. Zhu, C. M. Bergman, K. R. Thornton, D. Mittelman, and R. A. Gibbs. 2012. The Drosophila melanogaster genetic reference panel. Nature 482:173-178.

Mackay, T. F., E. A. Stone, and J. F. Ayroles. 2009. The genetics of quantitative traits: Challenges and prospects. Nat Rev Genet 10:565-577.

Macosko, E. Z., A. Basu, R. Satija, J. Nemesh, K. Shekhar, M. Goldman, I. Tirosh, A. R. Bialas, N. Kamitaki, E. M. Martersteck, J. J. Trombetta, D. A. Weitz, J. R. Sanes, A. K. Shalek, A. Regev, and S. A. McCarroll. 2015. Highly parallel genome-wide expression profiling of individual cells using nanoliter droplets. Cell 161:1202-1214.

Mali, P., L. Yang, K. M. Esvelt, J. Aach, M. Guell, J. E. DiCarlo, J. E. Norville, and G. M. Church. 2013. RNA-guided human genome engineering via Cas9. Science 339:823-826.

Maloof, J. N., J. O. Borevitz, T. Dabi, J. Lutes, R. B. Nehring, J. L. Redfern, G. T. Trainer, J. M. Wilson, T. Asami, C. C. Berry, D. Weigel, and J. Chory. 2001. Natural variation in light sensitivity of Arabidopsis. Nat Genet 29:441-446. 
Manuck, S. B. and J. M. McCaffery. 2014. Gene-environment interaction. Annu Rev Psychol 65:41-70.

Maranville, J. C., F. Luca, M. Stephens, and A. Di Rienzo. 2012. Mapping gene-environment interactions at regulatory polymorphisms: Insights into mechanisms of phenotypic variation. Transcription 3:56-62.

Masel, J. and M. L. Siegal. 2009. Robustness: Mechanisms and consequences. Trends Genet 25:395-403.

Matsui, T. and I. M. Ehrenreich. 2016. Gene-environment interactions in stress response contribute additively to a genotype-environment interaction. PLoS Genet 12:e1006158.

McKenna, A., G. M. Findlay, J. A. Gagnon, M. S. Horwitz, A. F. Schier, and J. Shendure. 2016. Whole-organism lineage tracing by combinatorial and cumulative genome editing. Science 353:aaf7907.

Mestek Boukhibar, L. and M. Barkoulas. 2016. The developmental genetics of biological robustness. Ann Bot 117:699-707.

Miki, D., W. Zhang, W. Zeng, Z. Feng, and J. K. Zhu. 2018. CRISPR/Cas9-mediated gene targeting in Arabidopsis using sequential transformation. Nat Commun 9:1967.

Mouradov, A., F. Cremer, and G. Coupland. 2002. Control of flowering time: Interacting pathways as a basis for diversity. Plant Cell 14(Suppl):S111-S130.

Myles, S., J. Peiffer, P. J. Brown, E. S. Ersoz, Z. Zhang, D. E. Costich, and E. S. Buckler. 2009. Association mapping: Critical considerations shift from genotyping to experimental design. Plant Cell 21:2194-2202.

Nadeau, J. H. and A. M. Dudley. 2011. Genetics. Syst Genet Sci 331:1015-1016.

Nijhout, H. F., F. Sadre-Marandi, J. Best, and M. C. Reed. 2017. Systems biology of phenotypic robustness and plasticity. Integr Comp Biol 57:171-184.

Nordborg, M., T. T. Hu, Y. Ishino, J. Jhaveri, C. Toomajian, H. Zheng, E. Bakker, P. Calabrese, J. Gladstone, R. Goyal, M. Jakobsson, S. Kim, Y. Morozov, B. Padhukasahasram, V. Plagnol, N. A. Rosenberg, C. Shah, J. D. Wall, J. Wang, K. Zhao, T. Kalbfleisch, V. Schulz, M. Kreitman, and J. Bergelson. 2005. The pattern of polymorphism in Arabidopsis thaliana. PLoS Biol 3:e196.

Paaby, A. B. and M. V. Rockman. 2014. Cryptic genetic variation: Evolution's hidden substrate. Nat Rev Genet 15:247-258.

Peter, J., M. De Chiara, A. Friedrich, J. X. Yue, D. Pflieger, A. Bergstrom, A. Sigwalt, B. Barre, K. Freel, A. Llored, C. Cruaud, K. Labadie, J. M. Aury, B. Istace, K. Lebrigand, P. Barbry, S. Engelen, A. Lemainque, P. Wincker, G. Liti, and J. Schacherer. 2018. Genome evolution across 1,011 Saccharomyces cerevisiae isolates. Nature 556:339-344.

Pfennig, D. W. 2021. Key questions about phenotypic plasticity. In D. W. Pfennig, ed., Phenotypic Plasticity and Evolution: Causes, Consequences, Controversies. CRC Press, Boca Raton, FL.

Pigliucci, M. 2001. Phenotypic Plasticity: Beyond Nature and Nurture. The John Hopkins University Press, Baltimore, MD.

Pritchard, J. K., M. Stephens, N. A. Rosenberg, and P. Donnelly. 2000. Association mapping in structured populations. Am J Hum Genet 67:170-181.

Queitsch, C., K. D. Carlson, and S. Girirajan. 2012. Lessons from model organisms: Phenotypic robustness and missing heritability in complex disease. PLoS Genet 8:e1003041.

Queitsch, C., T. A. Sangster, and S. Lindquist. 2002. Hsp90 as a capacitor of phenotypic variation. Nature 417:618-624.

Ragsdale, E. J. and N. A. Ivers. 2016. Specialization of a polyphenism switch gene following serial duplications in Pristionchus nematodes. Evolution 70:2155-2166.

Raj, B., D. E. Wagner, A. McKenna, S. Pandey, A. M. Klein, J. Shendure, J. A. Gagnon, and A. F. Schier. 2018. Simultaneous single-cell profiling of lineages and cell types in the vertebrate brain. Nat Biotechnol 36:442-450.

Rockman, M. V. 2008. Reverse engineering the genotype-phenotype map with natural genetic variation. Nature 456:738-744. 
Rockman, M. V. and L. Kruglyak. 2008. Breeding designs for recombinant inbred advanced intercross lines. Genetics 179:1069-1078.

Rosas, U., A. Cibrian-Jaramillo, D. Ristova, J. A. Banta, M. L. Gifford, A. H. Fan, R. W. Zhou, G. J. Kim, G. Krouk, K. D. Birnbaum, M. D. Purugganan, and G. M. Coruzzi. 2013. Integration of responses within and across Arabidopsis natural accessions uncovers loci controlling root systems architecture. Proc Natl Acad Sci USA 110:15133-15138.

Roy, K. R., J. D. Smith, S. C. Vonesch, G. Lin, C. S. Tu, A. R. Lederer, A. Chu, S. Suresh, M. Nguyen, J. Horecka, A. Tripathi, W. T. Burnett, M. A. Morgan, J. Schulz, K. M. Orsley, W. Wei, R. S. Aiyar, R. W. Davis, V. A. Bankaitis, J. E. Haber, M. L. Salit, R. P. St Onge, and L. M. Steinmetz. 2018. Multiplexed precision genome editing with trackable genomic barcodes in yeast. Nat Biotechnol 36:512-520.

Rutherford, S. L. and S. Lindquist. 1998. Hsp90 as a capacitor for morphological evolution. Nature 396:336-342.

Sadhu, M. J., J. S. Bloom, L. Day, J. J. Siegel, S. Kosuri, and L. Kruglyak. 2018. Highly parallel genome variant engineering with CRISPR-Cas9. Nat Genet 50:510-514.

Salome, P. A., K. Bomblies, R. A. Laitinen, L. Yant, R. Mott, and D. Weigel. 2011. Genetic architecture of flowering-time variation in Arabidopsis thaliana. Genetics 188:421-433.

Satija, R., J. A. Farrell, D. Gennert, A. F. Schier, and A. Regev. 2015. Spatial reconstruction of single-cell gene expression data. Nat Biotechnol 33:495-502.

Scheiner, S. M. 1993. Genetics and evolution of phenotypic plasticity. Annu Rev Ecol Syst 24:35-68.

Scheiner, S. M. and N. A. Levis. 2021. The loss of phenotypic plasticity via natural selection: Genetic assimilation. In D. W. Pfennig, ed., Phenotypic Plasticity and Evolution: Causes, Consequences, Controversies. CRC Press, Boca Raton, FL.

Schell, R., M. Mullis, and I. M. Ehrenreich. 2016. Modifiers of the genotype-phenotype map: Hsp90 and beyond. PLoS Biol 14:e2001015.

Schlichting, C. D. and M. Pigliucci. 1993. Control of phenotypic plasticity via regulatory genes. Am Nat 142:366-370.

Schlichting, C. D. and H. Smith. 2002. Phenotypic plasticity: Linking molecular mechanisms with evolutionary outcomes. Evol Ecol 16:189-211.

Schmalhausen, I. I. 1949. Factors of Evolution: The Theory of Stabilizing Selection. University of Chicago Press, Chicago.

Sharon, E., S. A. Chen, N. M. Khosla, J. D. Smith, J. K. Pritchard, and H. B. Fraser. 2018. Functional genetic variants revealed by massively parallel precise genome editing. Cell 175:544-557.e516.

Shindo, C., G. Bernasconi, and C. S. Hardtke. 2007. Natural genetic variation in Arabidopsis: Tools, traits and prospects for evolutionary ecology. Ann Bot 99:1043-1054.

Sieberts, S. K. and E. E. Schadt. 2007. Moving toward a system genetics view of disease. Mamm Genome 18:389-401.

Siegal, M. L. and J. Y. Leu. 2014. On the nature and evolutionary impact of phenotypic robustness mechanisms. Annu Rev Ecol Evol Syst 45:496-517.

Sieriebriennikov, B., N. Prabh, M. Dardiry, H. Witte, W. Roseler, M. R. Kieninger, C. Rodelsperger, and R. J. Sommer. 2018. A developmental switch generating phenotypic plasticity is part of a conserved multi-gene locus. Cell Rep 23:2835-2843.e2834.

Simpson, J. T. and M. Pop. 2015. The theory and practice of genome sequence assembly. Annu Rev Genomics Hum Genet 16:153-172.

Smith, E. N. and L. Kruglyak. 2008. Gene-environment interaction in yeast gene expression. PLoS Biol 6:e83.

Steinmetz, L. M., H. Sinha, D. R. Richards, J. I. Spiegelman, P. J. Oefner, J. H. McCusker, and R. W. Davis. 2002. Dissecting the architecture of a quantitative trait locus in yeast. Nature 416:326-330. 
Sultan, S. E. 2021. Phenotypic plasticity as an intrinsic property of organisms. In D. W. Pfennig, ed., Phenotypic Plasticity and Evolution: Causes, Consequences, Controversies. CRC Press, Boca Raton, FL.

Taylor, M. B. and I. M. Ehrenreich. 2014. Genetic interactions involving five or more genes contribute to a complex trait in yeast. PLoS Genet 10:e1004324.

Taylor, M. B. and I. M. Ehrenreich. 2015. Transcriptional derepression uncovers cryptic higher-order genetic interactions. PLoS Genet 11:e1005606.

Taylor, M. B., J. Phan, J. T. Lee, M. McCadden, and I. M. Ehrenreich. 2016. Diverse genetic architectures lead to the same cryptic phenotype in a yeast cross. Nat Commun 7:11669.

Ungerer, M. C., S. S. Halldorsdottir, M. D. Purugganan, and T. F. Mackay. 2003. Genotypeenvironment interactions at quantitative trait loci affecting inflorescence development in Arabidopsis thaliana. Genetics 165:353-365.

Via, S. and R. Lande. 1985. Genotype-environment interaction and the evolution of phenotypic plasticity. Evolution 39:505-522.

Waddington, C. H. 1953. Genetic assimilation of an acquired character. Evolution 7:118-126.

Wagner, D. E. and A. M. Klein. 2020. Lineage tracing meets single-cell omics: Opportunities and challenges. Nat Rev Genet 21:410-427.

Wagner, D. E., C. Weinreb, Z. M. Collins, J. A. Briggs, S. G. Megason, and A. M. Klein. 2018. Single-cell mapping of gene expression landscapes and lineage in the zebrafish embryo. Science 360:981-987.

Wei, X. and J. Zhang. 2017. The genomic architecture of interactions between natural genetic polymorphisms and environments in yeast growth. Genetics 205:925-937.

Weigel, D. 2012. Natural variation in Arabidopsis: From molecular genetics to ecological genomics. Plant Physiol 158:2-22.

Weigel, D. and M. Nordborg. 2005. Natural variation in Arabidopsis. How do we find the causal genes? Plant Physiol 138:567-568.

Weiss, C. V., J. I. Roop, R. K. Hackley, J. N. Chuong, I. V. Grigoriev, A. P. Arkin, J. M. Skerker, and R. B. Brem. 2018. Genetic dissection of interspecific differences in yeast thermotolerance. Nat Genet 50:1501-1504.

Werner, J. D., J. O. Borevitz, N. Warthmann, G. T. Trainer, J. R. Ecker, J. Chory, and D. Weigel. 2005. Quantitative trait locus mapping and DNA array hybridization identify an FLM deletion as a cause for natural flowering-time variation. Proc Natl Acad Sci USA 102:2460-2465.

West-Eberhard, M. J. 2003. Developmental Plasticity and Evolution. Oxford University Press, Oxford, UK.

Yadav, A., K. Dhole, and H. Sinha. 2016. Genetic regulation of phenotypic plasticity and canalisation in yeast growth. PLoS One 11:e0162326.

Yadav, A. and H. Sinha. 2018. Gene-gene and gene-environment interactions in complex traits in yeast. Yeast 35:403-416.

Yu, J., J. B. Holland, M. D. McMullen, and E. S. Buckler. 2008. Genetic design and statistical power of nested association mapping in maize. Genetics 178:539-551.

Zhao, K., M. J. Aranzana, S. Kim, C. Lister, C. Shindo, C. Tang, C. Toomajian, H. Zheng, C. Dean, P. Marjoram, and M. Nordborg. 2007. An Arabidopsis example of association mapping in structured samples. PLoS Genet 3:e4. 